\title{
Forecasting Macroeconomic Variables in a Small Open Economy: A Comparison between Small- and Large-Scale Models \\ Rangan Gupta* and Alain Kabundi**
}

\begin{abstract}
This paper compares the forecasting ability of five alternative models in predicting four key macroeconomic variables, namely, per capita growth rate, the Consumer Price Index (CPI) inflation, the money market rate, and the growth rate of the nominal effective exchange rate for the South African economy. Unlike the theoretical Small Open Economy New Keynesian Dynamic Stochastic General Equilibrium (SOENKDSGE), the unrestricted VAR, and the small-scale Bayesian Vector Autoregressive (BVAR) models, which are estimated based on four variables, the Dynamic Factor Model (DFM) and the large-scale BVAR models use information from a data-rich environment containing 266 macroeconomic time series observed over the period of 1983:01 to 2002:04. The results, based on Root Mean Square Errors (RMSEs), for one- to four-quarters-ahead out-of-sample forecasts over the horizon of 2003:01 to 2006:04, show that, except for the one-quarter-ahead forecast of the growth rate of the of nominal effective exchange rate, large-scale BVARs outperform the other four models consistently and, generally, significantly.
\end{abstract}

Journal of Economic Literature Classification: C11, C13, C33, C53.

Keywords: Small Open Economy New Keynesian Dynamic Stochastic Model, Dynamic Factor Model, VAR, BVAR, Forecast Accuracy.

\section{Introduction}

This paper compares the forecasting ability of five alternative models in predicting four key macroeconomic variables, namely, per capita growth rate, the Consumer Price Index (CPI) inflation, the money market rate, and the growth rate of the nominal effective exchange rate for an emerging market economy, which in our case happens to be South Africa. Specifically, we compare a standard Small Open Economy New Keynesian Dynamic Stochastic General Equilibrium (SOENKDSGE) model developed by Lubik and Schorfheide (2007), with two small-scale and two large-scale atheoretical models. The two-small scale models are the classical and Bayesian Vector Autoregressive (VAR) models involving only the above mentioned four key macroeconomic variables, while, the two large-scale models are the Dynamic Factor Model (DFM) and the large-scale Bayesian VAR (BVAR), both of which exploit information contained in a large cross-section of time series, 266 to be specific. ${ }^{1}$ Further note, within the small-scale and large-scale category of the BVARs, we estimate each of the models under five-alternative hyperparameter values specifying the prior. All the models are estimated over the period of 1983:01 to 2002:04 using quarterly data, ${ }^{2}$ and are then used to generate one- to fourquarters-ahead out-of-sample forecasts over a 16 quarters horizon of 2003:01 to 2006:04, with the performance of the models being compared using the Root Mean Square Error (RMSE) statistic.

The main motivation for this current piece of work is simple: We want to deduce which kind of standard model(s), theoretically grounded or atheoretical, small or large, is (are) best suited in forecasting an emerging market economy? To the best of our knowledge, this is the first attempt to look at such a wide array of small and large-scale models in forecasting a developing Small Open

\footnotetext{
* To whom correspondence should be addressed. Associate Professor, University of Pretoria, Department of Economics, Pretoria, 0002, South Africa, Email: Rangan.Gupta@up.ac.za. Phone: +27 12420 3460, Fax: +27 12 3625207.

** To whom correspondence should be addressed. Senior Lecturer, University of Johannesburg, Department of Economics, Johannesburg, 2006, South Africa, Email: akabundi@uj.ac.za. Phone: +27 11559 2061, Fax: +27 11 5593039.

${ }^{1}$ In general, the motivation to use a large data set to forecast an economy originates not only from the fact that such data is now available at lower cost, but also because, and, perhaps, more importantly, the increased power of computation has facilitated in using such huge amount of information to estimate and forecast with econometric models. In addition, central bankers, policymakers, and academics agree that economic agents monitor hundreds of economic variables in their decision-making process (Bernanke and Boivin, 2003).

${ }^{2}$ The choice of the in-sample period is driven by the recent paper by Ortiz and Sturzenegger (2007). In this paper, the authors use the Lubik and Schorfheide (2007) model to estimate an interest rate rule for South Africa over the period of 1983:01 to 2002:04. Given that the SOENKDSGE is estimated based on Bayesian methods, using the same estimation period allows us to use the same set of priors on the parameters of the model, as used by Ortiz and Sturzenegger (2007).
} 
Economy (SOE). At this stage, it must be emphasized that the choice of South Africa, as our country of interest, is purely data-driven, especially due to our requirement of information on 266 quarterly time series data, which, in turn, is derive from the existing studies of Gupta and Kabundi (2008a) and Das et al. (2008). Hence, there is no reason as to why similar research cannot be conducted for any other economy, given the general nature of the econometric models used here. Besides the obvious need of data, the choice of South Africa is also driven by the results of two recent papers, namely Liu et al. (2008) and Gupta and Kabundi (2008a), on forecasting the South African economy. Using a closed economy NKDSGE model, estimated using quarterly data over the period of 1970:01-2000:04, Liu et al (2008) forecasted growth per capita, inflation based on the Gross Domestic Product (GDP) deflator and the 91 days Treasury Bill rate for the period of 2001:01 to 2006:04. When the forecasts were compared with those generated from the Classical and Bayesian variants of the Vector Autoregression (VAR) models, the results indicated that the NKDSGE model outperformed both the Classical and the Bayesian VARs for inflation, but not for output growth and the nominal short-term interest rate. However, the differences in the RMSEs were not significant across the models. Gupta and Kabundi (2008a), used a DFM to forecast the same three variables over the same out-of-sample horizon, but based on an in-sample period of 1980:01 to 2000:04. Interestingly, when the forecast performance of the model was compared with an unrestricted VAR, alternative BVARs and the NKDSGE model developed by Liu et al. (2008), the authors found the DFM to outperform all the models in terms of forecasting the interest rate, while, it did no worse than the VAR and the BVARs in forecasting the other two variables. Gupta and Kabundi (2008a) attributed the better performance of the DFM to its ability to efficiently handle large amounts of information, which, in turn, contained information about the influence of foreign variables on the domestic economy, and, hence, its capability to forecast more accurately.

In such a backdrop, this paper should be viewed not only as an attempt to shed light on the type of model that is possibly best-suited for forecasting an emerging market, but also to check for the validity of the claim made by Gupta and Kabundi (2008a), by comparing the forecasts for the four variables obtained from the DFM, with that of a microfounded SOENKDSGE model and also large-scale BVAR models, of which the latter, based on their estimation method ${ }^{3}$ can also accommodate a panel as large as the one used in the DFM. ${ }^{4}$ For the sake of completeness, and as is standard in the forecasting literature, ${ }^{5}$ we also look at the predictive abilities of a small-scale VAR and small-scale BVARs. The remainder of the paper is organized as follows: Section 2 briefly discusses the SOENKDSGE, the DFM, the VAR and the Minnesota-type BVARs. Section 3 presents the results from the forecasting exercise and, finally, section 4 concludes.

\section{Alternative Forecasting Models:}

\subsection{The SOENKDSGE Model ${ }^{6}$ :}

The log-linearized version of the SOENKDSGE model, used in this paper, can be described by three main equations. The demand-side of the economy is characterized by an open economy IS curve as follows:

$y_{t}=\left\{\begin{array}{c}E_{t} y_{t+1}-[\tau+\alpha(2-\alpha)(1-\tau)]\left(R_{t}-E_{t} \pi_{t+1}\right)-\rho_{z} z_{t}(1) \\ -\alpha[\tau+\alpha(2-\alpha)(1-\tau)] E_{t} \Delta q_{t+1}+\alpha(2-\alpha) \frac{1-\tau}{\tau} E_{t} \Delta y_{t+1}^{*}\end{array}\right\}$

where, $y_{t}$ : real GDP; $R_{t}$ : nominal money market rate; $\pi_{t}:$ CPI inflation; $z_{t}$ : growth rate of a nonstationary world technology process $Z_{t} ; q_{t}$ : Terms of Trade (TOT, relative price of exports to imports); $y_{t}^{*}$ : exogenous world output; $\tau$ : elasticity of intertemporal substitution; $\alpha$ : import share; $\rho_{z}$ : AR coefficient of $z_{t}$. To ensure stationarity, all real variables are expressed as percentage deviations from $Z_{t}$.

While, the supply-side of the economy is captured by an open economy Phillips curve:

\footnotetext{
${ }^{3}$ See Section 2 for details regarding the estimation of BVARs.

${ }^{4}$ See De Mol et al. (2006) and Gupta and Kabundi (2008b) for two recent studies that deals with the comparison of the forecast performances between large-scale BVARs and DFM.

${ }^{5}$ See Dua and Ray (1995), LeSage (1999), Gupta and Sichei (2006), Gupta (2006, 2007a,b) and Gupta and Das (2008) for further details.

${ }^{6}$ See Gali and Monacelli (2005), Lubik and Schorfheide (2007) and Ortiz and Sturzenegger (2007) for derivations of the model equations.
} 
$\pi_{t}=\beta E_{t} \pi_{t+1}+\alpha \beta E_{t} \Delta q_{t+1}-\alpha \Delta q_{t}+\frac{\kappa}{\tau+\alpha(2-\alpha)(1-\tau)}\left(y_{t}-\overline{y_{t}}\right)$

where, $\overline{y_{t}}=-\alpha(2-\alpha) \frac{1-\tau}{\tau} y_{t}^{*}$ : potential output (in absence of nominal rigidities); $\beta$ : discount factor, and; $\kappa$ : the structural parameter defining the slope of the Phillips curve.

Finally, the monetary authority's reaction function is determined by an interest rate-rule of the following nature:

$R_{t}=\rho_{R} R_{t-1}+\left(1-\rho_{R}\right)\left[\psi_{1} \pi_{t}+\psi_{2} y_{t}+\psi_{3} \Delta s_{t}\right]+\varepsilon_{t}^{R} ; \varepsilon_{t}^{R} \sim N\left(0, \sigma_{R}^{2}\right)$

where, $\rho_{R}$ : persistence in the interest rate; $s_{t}$ : nominal effective exchange rate; $\psi_{i}, i=1,2,3$ : monetary authority's reaction parameter to inflation, output and exchange rate fluctuations, respectively.

The exchange rate is introduced via the CPI inflation according to:

$\pi_{t}=\Delta s_{t}+(1-\alpha) \Delta q_{t}+\pi_{t}^{*}$

where, $\pi_{t}^{*}$ : world inflation shock. Growth rate in the TOT, in turn, follows an exogenous AR(1) process:

$\Delta q_{t}=\rho_{q} \Delta q_{t-1}+\varepsilon_{t}^{q} ; \varepsilon_{t}^{q} \sim N\left(0, \sigma_{q}^{2}\right)$

As does the world output and inflation:

$y_{t}^{*}=\rho_{y^{*}} y_{t-1}^{*}+\varepsilon_{t}^{y^{*}} ; \varepsilon_{t}^{y^{*}} \sim N\left(0, \sigma_{y^{*}}^{2}\right)$
$\pi_{t}^{*}=\rho_{\pi^{*}} \pi_{t-1}^{*}+\varepsilon_{t}^{\pi^{*}} ; \varepsilon_{t}^{\pi^{*}} \sim N\left(0, \sigma_{\pi^{*}}^{2}\right)$

The SOENKDSGE model presented above is estimated using Bayesian methods. The object of interest is the vector of parameters ${ }^{7}$ :

$\theta=\left(\psi_{1}, \psi_{2}, \psi_{3}, \alpha, \beta, \kappa, \tau, \rho_{q}, \rho_{z}, \rho_{y^{*}}, \rho_{\pi^{*}}, \rho_{R}, \sigma_{R}, \sigma_{q}, \sigma_{z}, \sigma_{y^{*}}, \sigma_{\pi^{*}}\right)$

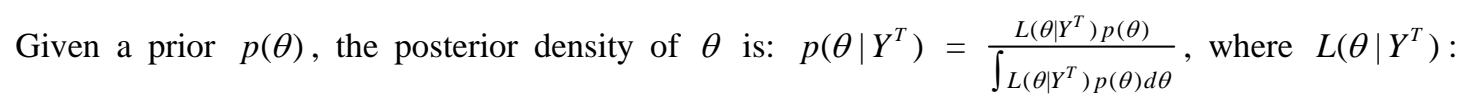
likelihood conditional on observed data $Y^{T}=Y_{1} \ldots \ldots, Y_{T}$.

In our case, $Y_{t}=\left[\Delta y_{t}+z_{t}, 4 \pi_{t}, 4 R_{t}, \Delta s_{t}, \Delta q_{t}\right]^{\prime} . \quad L\left(\theta \mid Y^{T}\right)$ is computed by combining the state-space representation obtained from the Rational expectations solution of the model and the Kalman filter, based on normally distributed errors, with the posterior draws being obtained using the Markov Chain Monte Carlo methods from MCMC method. After obtaining the mode of the posterior, a Random Walk Metropolis algorithm is used to generate posterior draws. Then, from the generated values, we obtain the point estimates and measures of uncertainty for $\theta$. Finally, once we are done estimating over the in-sample period of 1983:01-2002:04, we recursively estimate over 2003:01-2006:04 to generate oneto four-quarters ahead forecasts.

\subsection{The DFM}

This study uses the Dynamic Factor Model (DFM) developed by Forni et al. (2005) to extract common components between macroeconomics series, and then these common components are used to forecast four key macro variables of South Africa. In the VAR models, since all variables are used in forecasting, the number of parameters to estimate depend on the number of variables $n$. With such a large information set, $n$, the estimation of a large number of parameters leads to a curse of dimensionality. The DFM uses information set accounted by few factors $q<<n$, which transforms the curse of dimensionality into a blessing of dimensionality.

The DFM expresses individual times series as the sum of two unobserved components: a common component driven by a small number of common factors and an idiosyncratic component, which are specific to each variable. The relevance of the method is that the DFM is able to extract the few factors that explain the comovement of all South African macroeconomic variables. Forni et al. (2005) demonstrated that when the number of factors is small relative to the number of variables and the panel

\footnotetext{
${ }^{7}$ See Subsection 5.2.3 in Ortiz and Sturzenegger (2007) for the specification of the priors for these parameters.
} 
is heterogeneous, the factors can be recovered from the present and past observations.

Consider an $n \times 1$ covariance stationary process $Y_{t}=\left(y_{1 t}, \ldots, y_{n t}\right)^{\prime}$. Suppose that $X_{t}$ is the standardized version of $Y_{t}$, i.e. $X_{t}$ and has a mean zero and a variance equal to one. Under the DFM proposed by Forni et al. (2005), $X_{t}$ is described by a factor model, it can be written as the sum of two orthogonal components:

$$
x_{i t}=b_{i}(L) f_{t}+\xi_{i t}=\lambda_{i} F_{t}+\xi_{i t}=\chi_{i t}+\xi_{i t}
$$

or, in vector notation:

$$
X_{t}=B(L) f_{t}+\xi_{i t}=\Lambda F_{t}+\xi_{i t}=\chi_{i t}+\xi_{i t}
$$

where $f_{t}$ is a $q \times 1$ vector of dynamic factors, $B(L)=B_{0}+B_{1} L+\ldots+B_{s} L^{s}$ is an $n \times q$ matrix of factor loadings of order $s, \xi_{i t}$ is an $n \times 1$ vector of idiosyncratic components, $F_{t}$ is $r \times 1$ vector of factors, with $r=q(s+1)$. However, in a more general framework $r \geq q$, instead of the more restrictive $r=q(s+1)$. In a DFM, $f_{t}$ and $\xi_{\text {it }}$ are mutually orthogonal stationary processes, while, $\chi_{i t}$ is the common component.

In factor analysis jargon, $X_{t}=B(L) f_{t}+\xi_{i t}$ is referred to as dynamic factor model, and $X_{t}=\Lambda F_{t}+\xi_{i t}$ is the static factor model. Similarly, $f_{t}$ is regarded as vector of dynamic factors, while, $F_{t}$ is the vector of static factors. Since dynamic common factors are latent, they need to be estimated. Forni et al. (2005) estimate the dynamic factors through the use of dynamic principal component analysis. It involves the estimation of the eigenvalues and eigenvectors decomposition of the spectral density matrix of $X_{t}$, which is a generalization of the orthogonalization process in case of static principal components. ${ }^{8}$ The DFM of Forni et al. (2005) is estimated in two steps to solve the end-ofsample problems caused by two-sided filtering encountered with the Dynamic Principle Component Analysis (DPCA) used in Forni et al. (2000). Due to end-of-sample problems, this method is not suited for forecasting. Firstly, the DPCA is used to compute estimates of covariance matrices of common and idiosyncratic components of $X_{t}$ at all leads and lags as inverse Fourier transforms of the corresponding estimated spectral density matrices. Thus, the spectral density matrix of $X_{t}$ is given by $\Sigma(\theta)=\Sigma_{\chi}(\theta)+\Sigma_{\xi}(\theta)$. Secondly, these estimates are used in the construction of $r$ linear combinations of the observations having smallest idiosyncratic-common variance ratio.

\subsection{The VAR and BVARs ${ }^{9}$}

The Vector Autoregressive (VAR) model, though 'atheoretical', is particularly useful for forecasting purposes. An unrestricted VAR model, as suggested by Sims (1980), can be written as follows:

$y_{t}=A_{0}+A(L) y_{t}+\varepsilon_{t}$

where $y$ is a $(n \times 1)$ vector of variables being forecasted; $A(L)$ is a $(n \times n)$ polynomial matrix in the backshift operator $L$ with lag length $p$, i.e., $A(L)=A_{1} L+A_{2} L^{2}+\ldots \ldots \ldots \ldots \ldots . .+A_{p} L^{p} ; A_{0}$ is a $(n \times 1)$ vector of constant terms, and $\varepsilon$ is a $(n \times 1)$ vector of error terms. In our case, we assume that $\varepsilon \sim N\left(0, \sigma^{2} I_{n}\right)$, where $I_{n}$ is a $n \times n$ identity matrix.

Note the VAR model, generally uses equal lag length for all the variables of the model. One drawback of VAR models is that many parameters need to be estimated, some of which may be insignificant. This problem of overparameterization, resulting in multicollinearity and a loss of degrees of freedom, leads to inefficient estimates and possibly large out-of-sample forecasting errors. One solution, often adapted, is simply to exclude the insignificant lags based on statistical tests. Another approach is to use a near VAR, which specifies an unequal number of lags for the different equations.

\footnotetext{
${ }^{8}$ See Gupta and Kabundi (2008a) for a detailed description of the model.

${ }^{9}$ This section relies heavily on the discussion available on VAR and BVAR in Dua and Ray (1995), LeSage

(1999), Gupta and Sichei (2006), Gupta (2006, 2007, 2008) and Gupta and Das (2008).
} 
However, an alternative approach to overcoming this overparameterization, as described in Litterman (1981), Doan et al. (1984), Todd (1984), Litterman (1986), and Spencer (1993), is to use a BVAR model. Instead of eliminating longer lags, the Bayesian method imposes restrictions on these coefficients by assuming that they are more likely to be near zero than the coefficients on shorter lags. However, if there are strong effects from less important variables, the data can override this assumption. The restrictions are imposed by specifying normal prior distributions with zero means and small standard deviations for all coefficients with the standard deviation decreasing as the lags increase. The exception to this is that the coefficient on the first own lag of a variable has a mean of unity. Litterman (1981) used a diffuse prior for the constant. This is popularly referred to as the 'Minnesota prior' due to its development at the University of Minnesota and the Federal Reserve Bank at Minneapolis.

Formally, as discussed above, the means and variances of the Minnesota prior take the following form:

$$
\beta_{i} \sim N\left(1, \sigma_{\beta_{i}}^{2}\right) \text { and } \beta_{j} \sim N\left(0, \sigma_{\beta_{j}}^{2}\right)
$$

where $\beta_{i}$ denotes the coefficients associated with the lagged dependent variables in each equation of the VAR, while $\beta_{j}$ represents any other coefficient. In the belief that lagged dependent variables are important explanatory variables, the prior means corresponding to them are set to unity. However, for all the other coefficients, $\beta_{j}$ 's, in a particular equation of the VAR, a prior mean of zero is assigned to suggest that these variables are less important to the model.

The prior variances $\sigma_{\beta_{i}}^{2}$ and $\sigma_{\beta_{j}}^{2}$, specify uncertainty about the prior means $\bar{\beta}_{i}=1$, and $\bar{\beta}_{j}=0$, respectively. Because of the overparameterization of the VAR, Doan et al. (1984) suggested a formula to generate standard deviations as a function of small numbers of hyperparameters: $w, d$, and a weighting matrix $f(i, j)$. This approach allows the forecaster to specify individual prior variances for a large number of coefficients based on only a few hyperparameters. The specification of the standard deviation of the distribution of the prior imposed on variable $j$ in equation $i$ at lag $m$, for all $i, j$ and $m$, defined as $S_{1}(i, j, m)$, can be specified as follows:

$S_{1}(i, j, m)=[w \times g(m) \times f(i, j)] \frac{\hat{\sigma}_{j}}{\hat{\sigma}_{i}}$

with $f(i, j)=1$, if $i=j$ and $k_{i j}$ otherwise, with $\left(0 \leq k_{i j} \leq 1\right), g(m)=m^{-d}, d>0$. Note that $\hat{\sigma}_{i}$ is the estimated standard error of the univariate autoregression for variable $i$. The ratio $\hat{\sigma}_{i} / \hat{\sigma}_{j}$ scales the variables to account for differences in the units of measurement and, hence, causes specification of the prior without consideration of the magnitudes of the variables. The term $w$ indicates the overall tightness and is also the standard deviation on the first own lag, with the prior getting tighter as we reduce the value. The parameter $g(m)$ measures the tightness on lag $m$ with respect to lag 1 , and is assumed to have a harmonic shape with a decay factor of $d$, which tightens the prior on increasing lags. The parameter $f(i, j)$ represents the tightness of variable $j$ in equation $i$ relative to variable $i$, and by increasing the interaction, i.e., the value of $k_{i j}$, we can loosen the prior. ${ }^{10}$ Note, in the standard Minnesota-type prior, the overall tightness ( $w$ ) takes the values of $0.1,0.2$ and 0.3 , while, the lag decay $(d)$ is generally chosen to be equal to $0.5,1.0$ and 2.0. The interaction parameter $\left(k_{i j}\right)$ is traditionally set at $=0.5$. The small-scale BVARs would be estimated with this set of parameterization of the priors.

Given that, we have domestic as well as foreign and world variables within the 266 data series used for the large-scale models, and realizing that South Africa is a small open economy, and, hence, domestic variables would have minimal, if any, effect on foreign and world variables, while, the latter set of variables is sure to have an influence on the South African variables, setting $k_{i j}=0.5$ could be quite far fetched from reality. Hence, borrowing from the BVAR models used for regional forecasting, involving both regional and national variables, and following Kinal and Ratner (1986), Shoesmith (1992) and Gupta and Kabundi (2008b), the weight of a foreign or world variable in a foreign or world equation, as well as a domestic equation, is set at 0.6. The weight of a domestic variable in other domestic equation is fixed at 0.1 and that in a foreign or world equation at 0.01 . Finally, the weight of

\footnotetext{
${ }^{10}$ For an illustration, see Dua and Ray (1995).
} 
the domestic variable in its own equation is 1.0. These weights are in line with Litterman's circle-star structure. Star (foreign or world) variables affect both star and circle (domestic) variables, while, circle variables primarily influence only other circle variables. ${ }^{11}$ Clearly then, the large-scale BVARs are estimated with asymmetric priors.

Finally, once the priors have been specified, the alternative BVARs, whether based on the 4 variables or all of the 266 variables, are estimated using Theil's (1971) mixed estimation technique. Specifically, suppose we denote a single equation of the VAR model as: $y_{1}=X \beta+\varepsilon_{1}$, with $\operatorname{Var}\left(\varepsilon_{1}\right)=\sigma^{2} I$, then the stochastic prior restrictions for this single equation can be written as:

$$
\left[\begin{array}{c}
M_{111} \\
M_{112} \\
\cdot \\
\cdot \\
\cdot \\
M_{n n m}
\end{array}\right]=\left[\begin{array}{cccccc}
\sigma / \sigma_{111} & 0 & . & . & . & 0 \\
0 & \sigma / \sigma_{112} & 0 & . & . & 0 \\
\cdot & \cdot & . & . & . & \cdot \\
\cdot & . & . & . & . & \cdot \\
0 & . & . & . & . & 0 \\
0 & 0 & . & . & 0 & \sigma / \sigma_{n n m}
\end{array}\right]\left[\begin{array}{c}
a_{111} \\
a_{112} \\
\cdot \\
\cdot \\
\cdot \\
a_{n n m}
\end{array}\right]+\left[\begin{array}{c}
u_{111} \\
u_{112} \\
\cdot \\
\cdot \\
\cdot \\
u_{n n m}
\end{array}\right]
$$

Note, $\operatorname{Var}(u)=\sigma^{2} I$ and the prior means $M_{i j m}$ and $\sigma_{i j m}$ take the forms shown in (11) and (12). With (13) written as:

$$
r=R \beta+u
$$

and the estimates for a typical equation are derived as follows:

$$
\hat{\beta}=\left(X^{\prime} X+R^{\prime} R\right)^{-1}\left(X^{\prime} y_{1}+R^{\prime} r\right)
$$

Essentially then, the method involves supplementing the data with prior information on the distribution of the coefficients. The number of observations and degrees of freedom are increased by one in an artificial way, for each restriction imposed on the parameter estimates. The loss of degrees of freedom due to over-parameterization associated with a classical VAR model is, therefore, not a concern in the BVARs.

\section{Data}

While, the SOENKDSGE and the small-scale VAR and BVARs, includes data on only the four variables of interest, namely, per capita growth rate, the Consumer Price Index (CPI) inflation, the money market rate, and the growth rate of the nominal effective exchange rate, the DFM and the largescale BVARs includes 266 quarterly series ${ }^{12}$ of South Africa, covering the real, nominal, and financial sectors. We also have intangible variables, such as confidence indices, and survey variables. In addition to national variables, the paper uses a set of global variables such as commodity industrial inputs price index and crude oil prices. The data also comprises series of major trading partners such as Germany, the United Kingdom (UK), and the United States (US) of America. The in-sample period contains data from 1983Q1 to 2002Q4, while the out-of-sample set is 2001Q1-2006Q4. All series are seasonally adjusted and made covariance stationary. The more powerful DFGLS test of Elliott, Rothenberg, and Stock (1996), instead of the most popular, but low in power, ADF test, is used to assess the degree of integration of all series. All nonstationary series are made stationary through differencing. The Schwarz information criterion is used in the selecting the appropriate lag length in such a way that no serial correction is left in the stochastic error term. Where there were doubts about the presence of unit root, the KPSS test proposed by Kwiatowski, Phillips, Schmidt, and Shin (1992), with the null hypothesis of stationarity, was applied. All series are standardized to have a mean of zero and a constant variance. It must, however, be pointed out that, non-stationarity is not an issue with the BVAR, since Sims et al. (1990) indicates that with the Bayesian approach entirely based on the likelihood function, the associated inference does not need to take special account of nonstationarity, since the likelihood function has the same Gaussian shape regardless of the presence of nonstationarity. Hence, for the sake

\footnotetext{
${ }^{11}$ We also experimented by assigning higher and lower interaction values, in comparison to those specified above, to the star variables in both the star and circle equations, but, the rank ordering of the alternative forecasts remained the same.

${ }^{12}$ The data set contains a total of 245 series of South Africa , two global variables, and 19 series of major trading partners. Details about data and their statistical treatment are available upon request. Details about data and their statistical treatment of the variables used to estimate the DFM are available upon request.
} 
of comparison amongst the VARs, both classical and Bayesian, we make no attempt o make the variables stationary, unlike in the DFM. ${ }^{13}$ Note as far as the SOENKDSGE is concerned, we follow Lubik and Schorfheide (2007) and Ortiz and Sturzenegger (2007), in pre-filtering the data, by demeaning them using their sample means.

There are various statistical approaches in determining the number of factors in the DFM. For example, Bai and Ng (2002) developed an information criteria, based on AIC and BIC, guiding the selection of the number of factors in large dimensional panels. The principal component analysis (PCA) can also be used in establishing the number of factors in the DFM. The PCA suggests that the selection of a number of factors $q$ be based on the first eigenvalues of the spectral density matrix of $X_{t}$. Then, the principal components are added until the increase in the explained variance is less than a specific $\alpha=0.05$. The Bai and $\mathrm{Ng}$ (2002) approach proposes five static factors, while Bai and $\mathrm{Ng}$ (2007) suggests two primitive or dynamic factors. Similar to the latter method, the principal component technique, as proposed by Forni et al. (2000), suggests two dynamic factors. The first two dynamic principal components explain approximately 99 percent of variation, while the eigenvalue of the third component is $0.005<0.05$.

\section{Evaluation of Forecast Accuracy}

Given the specifications of the models, we estimate the five alternative models, namely, the SOENKDSGE, the DFM, VAR, small-scale BVARs and the large-scale BVARs over the period of 1983:1 to 2002:04, based on quarterly data. Then we compute the out-of-sample one- through fourquarters-ahead forecasts for the period of 2003:01 to 2006:04, and compare the forecast accuracy of the five alternative models with respect to each other. The different types of the VARs are estimated with 5 $\operatorname{lags}^{14}$ of each variable. Since we use five lags, the initial five quarters of the sample, 1983:01 to 1984:01, are used to feed the lags. We generate dynamic forecasts, as would naturally be achieved in actual forecasting practice. The models are re-estimated each quarter over the out-of-sample forecast horizon in order to update the estimate of the coefficients, before producing the 4-quarters-ahead forecasts. This iterative estimation and 4-steps-ahead forecast procedure was carried out for 16 quarters, with the first forecast beginning in 2003:01. This experiment produced a total of 16 onequarter-ahead forecasts, 16-two-quarters-ahead forecasts, and so on, up to 16 4-step-ahead forecasts. The RMSEs ${ }^{15}$ for the 16 , quarter 1 through quarter 4 forecasts are then calculated for the per capita growth, CPI inflation, the money market rate and the growth rate of the effective nominal exchange rates. The values of the RMSE statistic for one- to four-quarters -ahead forecasts for the period 2003:01 to 2006:04 are then examined. The model that produces the lowest average value for the RMSE is selected, as the 'optimal' model for a specific variable.

In Tables 1 to 4, we compare the RMSEs of one- to four-quarters-ahead out-of-sample-forecasts for the period of 2003:01 to 2006:04, generated by the abovementioned models. At this stage, a few words need to be said regarding the choice of the evaluation criterion for the out-of-sample forecasts generated from Bayesian models. As Zellner (1986) points out the “optimal” Bayesian forecasts will differ depending upon the loss function employed and the form of predictive probability density function". In other words, Bayesian forecasts are sensitive to the choice of the measure used to evaluate the out-of-sample forecast errors. However, Zellner (1986) points out that the use of the mean of the predictive probability density function for a series, is optimal relative to a squared error loss function and the Mean Squared Error (MSE), and, hence, the RMSE is an appropriate measure to evaluate performance of forecasts, when the mean of the predictive probability density function is used. This is exactly what we do below in Tables 1 through 4, when we use the average RMSEs over the one- to

\footnotetext{
${ }^{13}$ See Dua and Ray (1995) for further details.

${ }^{14}$ The choice of 5 lags is based on the unanimity of the sequential modified LR test statistic, Akaike information criterion (AIC), the final prediction error (FPE) criterion and the Hannan-Quinn (HQ) information criterion applied to a stable VAR estimated with the four variables of concern. Note, stability, as usual, implies that no roots were found to lie outside the unit circle.

${ }^{15}$ Note that if $A_{t+n}$ denotes the actual value of a specific variable in period $t+n$ and ${ }_{t} F_{t+n}$ is the forecast made in period $t$ for $t+n$, the RMSE statistic can be defined as: $\sqrt{\frac{1}{N} \sum\left(A_{t+n}-{ }_{t} F_{t+n}\right)}$. For $n=1$, the summation runs from
} 2003:01 to 2006:04, and for $n=2$, the same covers the period of 2003:02 to 2006:04, and so on. 
four-quarter-ahead forecasting horizon. The conclusions, regarding each of the four variables, based on the average one- to four-quarters-ahead RMSEs, from these tables can be summarized as follows:

Per Capita Growth Rate: Based on the average RMSEs for one- to four-quarters-ahead forecasts, all the athoretical models outperform the SOENKDSGE model. However, within the category of the atheoretical models, it is the large-scale BVAR model with $w=$ $0.2, d=2$ that tends to stand out for each of the quarters. CPI Inflation: Though the SOENKDSGE model performs better than the DFM, it, in turn, is outperformed by not only the small-scale VARs, both classical and Bayesian, but also by the large-scale BVARs. As with per capita growth rate, the best performing model, both in terms of average RMSEs and each of the quarters ahead, is a large-scale BVAR, but with a relatively loose prior of $w=0.3, d=0.5$.

(iii) Money Market Rate: For the money market rate, the pattern of the results are exactly the same as with the per capita growth rate. Specifically, all the atheoretical models outperforms the SOENKDSGE, with the large-scale BVAR based on $w=0.2, d=2$, being the best performer amongst the five alternative types of models used for forecasting in terms of the RMSEs for at each of the one- to four-quarters-ahead forecasts, and, hence, also on average.

(iv) Growth Rate of Nominal Effective Exchange Rate: Unlike in the case of the other three variables, the SOENKDSGE is the best performing model in case of predicting the growth rate of the nominal effective exchange rate. The small-scale BVAR with $w=0.1 d$ $=1$ and the large-scale BVAR with $w=0.2, d=2$ comes in as the second and third best performing models respectively, based on the average RMSEs for one- to four-quartersahead forecasts. A closer look, however indicates that the large-scale BVAR with $w=$ $0.2, d=2$ outperforms the SOENKDSGE and the "optimal” small-scale BVAR for all the quarters, except the first.

To put these results into perspective, we draw comparison to the results of Gupta and Kabundi (2008a). The authors found the DFM to outperform the closed-economy NKDSGE model, the small-scale VAR and the BVAR models in terms of forecasting the interest rate, while, it did no worse than the VAR and the BVARs in forecasting the per capita growth rate and the inflation rate. Here, however, the SOENKDSGE model is found to outperform the DFM in terms of forecasting the exchange rate and the CPI inflation. So, when compared to Gupta and Kabundi (2008) open economy assumptions does help in improving the forecast performances of a microfounded model, at least for the CPI inflation and the growth rate of the nominal effective exchange rate. Moreover, the DFM is also outperformed by the small and large-scale VARs for all the four variables. Overall, the current study obtains results in favor of large-scale BVAR models, pointing to the fact that information contained in data-rich environment, which also allows to incorporate asymmetric effects of foreign variables on domestic variables, help to enhance the prediction of macroeconomic variables in emerging market countries.

An important question is, whether the differences we notice in the forecasting performances, based on the RMSEs, are indeed statistically significant. To answer this question, we compute the across model tests of forecast accuracy proposed by Diebold and Mariano (1995). Given that there are five alternative models, we use a parsimonious approach while reporting the Diebold-Mariano (1995) test statistic. ${ }^{16}$ We compare each of the one- to four-quarters-ahead forecasts generated by the best or "optimal" model, i.e. the model with the lowest average RMSE, to those of the second best model. The exception to this rule, is, however, the case of the growth rate of the nominal effective exchange rate. In this case, we compare the best three models. The reason being that we would like to see how the performance of the large-scale BVAR, which produces the third best average RMSEs in this case even

\footnotetext{
${ }^{16}$ The test statistic is defined as follows: For instance, let $\left\{e_{t}^{b}\right\}_{t=1}^{T}$ denote the associated forecast errors from the best model and $\left\{e_{t}^{s b}\right\}_{t=1}^{T}$ denote the forecast errors from the alternative, second-best model. The test statistic is then defined as $s=\frac{l}{\sigma_{l}}$, where $l$ is the sample mean of the "loss differentials", $\left\{l_{t}\right\}_{t=1}^{T}$, using $l_{t}=\left(e_{t}^{b}\right)^{2}-\left(e_{t}^{s b}\right)^{2}$ for all $t=1,2,3, \ldots, T$, and where $\sigma_{l}$ is the standard error of $l$. The $s$ statistic is asymptotically distributed as a standard normal random variable and can be estimated under the null hypothesis of equal forecast accuracy, i.e. $l=0$. Therefore, a negative value of $s$ would suggest that the best model outperforms the alternative model in terms of out-of-sample forecasting.
} 
though being the overwhelming favorite in the other three cases, compares with two best models. As can be seen from Table 5, in case of the per capita growth rate, the large-scale BVAR with $w=0.2, d=$ 2 outperforms the next-best model (the small-scale BVAR with $w=0.3, d=0.5$ ), with the Diebold and Mariano (1995) test statistics being significant at the 1 percent level for all the four quarters. Similarly, the Diebold and Mariano (1995) test statistics for the CPI inflation reveal that the large-scale BVAR with $w=0.3, d=0.5$ performs significantly better than the second best model, namely, the small-scale BVAR with $w=0.3, d=0.5$. However, for the money market rate, the performance of the large-scale BVAR with $w=0.3, d=0.5$ is statically superior to that of the small-scale BVAR with $w=0.1, d=2$ only for the first three quarters. The picture is different for the growth rate of the nominal effective exchange rate, where in the first quarter the large-scale BVAR with $w=0.2, d=2$ is outperformed significantly by both the small-scale BVAR with $w=0.1, d=1$ and the SOENKDSGE. However, for the next three quarters, even though the large-scale BVAR outperforms the other two models, the test statistic is significant only for the second quarter-ahead-forecast.

\section{Conclusions}

This paper compares the forecasting ability of five alternative models in predicting per capita growth rate, the Consumer Price Index (CPI) inflation, the money market rate, and the growth rate of the nominal effective exchange rate for South Africa. Specifically, we compare a standard SOENKDSGE model with two small-scale and two large-scale atheoretical models. The two-small scale models are the classical and Bayesian VAR models involving only the above mentioned four key macroeconomic variables, while, the two large-scale models are the DFM and the large-scale BVAR, both of which exploit information contained in a large cross-section of time series. All the models are estimated over the period of 1983:01 to 2002:04 using quarterly data, and are then used to generate one- to fourquarters-ahead out-of-sample forecasts over a 16 quarters horizon of 2003:01 to 2006:04. When the performance of the models are compared using the Root Mean Square Error (RMSE) statistic, our results indicate that data-rich large-scale BVARs are better suited in forecasting the key macroeconomic variables relative to the small-scale models involving only the few variables of interest.

However, it is important to point out that, there are at least two major limitations to using a Bayesian approach for forecasting. Firstly, as it is clear from Tables 1 to 4 , the forecast accuracy is sensitive to the choice of the priors. So if the prior is not well specified, an alternative model used for forecasting may perform better. Secondly, in case of the Bayesian models, one requires to specify an objective function, for example the average RMSEs, to search for the 'optimal' priors, which, in turn, needs to be optimized over the period for which we compute the out-of-sample forecasts. However, there is no guarantee that the chosen parameter values specifying the prior will continue to be 'optimal' beyond the period for which it was selected. Nevertheless, the importance of BVARs cannot be ignored, especially when one realizes that they can accommodate large number of time series, and in reality economic agents do monitor hundreds of economic variables in their decision-making process.

But, it is also important to check for the robustness of our conclusions, by redoing the exercise with BVARs based on alternative forms of priors, other than the Minnesota-type used in this paper. In this regard, a good starting point would be to use the double exponential priors as in De Mol et al. (2006). In addition to this, one might want to revisit the forecast performances of the BVARs by assuming a more general error structure, as in Gupta (2007), to account for non-constant variance of the variables, and, also look at Bayesian Vector Error Correction Models (BVECMs). As pointed out by LeSage (1990), Gupta (2006, 2008) and Zita and Gupta (2008), even though non-stationarity is not an issue with the Bayesian approach BVECMs, in general, tends to outperform BVARs, since Error Correction Models (ECMs) use long-run equilibrium relationships from economic theory to explain short-run dynamics of data. Finally, given that the large-scale BVAR, just like the other atheoretical models used in this paper, is not immune to the "Lucas Critique", ${ }^{17}$ however capable it might be in handling largenumber of variables, one would want to develop a more modified SOENKDSGE model, which, in turn, would allow for habit persistence, wage rigidity, imperfect pass-through, and import price rigidity, amongst other things.

\footnotetext{
${ }^{17}$ See Lucas (1976) for further details.
} 


\section{References}

Bai J and Ng S. Determining the Number of Factors in Approximate Factor Models. Econometrica 2002; 70: 191-221.

Bai J and Ng S. Determining the Number of Primitive Shocks in Factor Models. Journal of Business and Economic Statistics 2007; 25: 52-60.

Bernanke B and Boivin J. Monetary Policy in a Data-Rich Environment. Journal of Monetary Economics 2003; 50: 525-546.

Burger P and Van Rensburg LJ. Metropolitan House Prices in South Africa: Do they Converge? South African Journal of Economics 2008; 76:291-297.

Chamberlain G. Funds, Factors, and Diversification in Arbitrage Pricing Models. Econometrica 1983; 51: 1281-1304.

Chamberlain G and Rothschild M. Arbitrage, Factor Structure and Mean-Variance Analysis in Large Markets. Econometrica 1983; 51: 1305-1324.

Cristadoro R, Forni M, Reichlin L, and Giovanni V. A Core Inflation Indicator for the Euro Area. Journal of Money, Credit and Banking 2005; 37: 539-560.

Das S, Gupta R and Kabundi A. Is a DFM Well-Suited for Forecasting Regional House Price Inflation? Working Paper 2008; 200814, University of Pretoria, Department of Economics.

De Mol C, Giannone D, and Reichlin L. Forecasting Using a Large Number of Predictors: Is Bayesian Regression a Valid Alternative to Principal Components? CEPR Discussion Papers 2006; No. 5829.

Doan TA, Litterman RB and Sims CA. Forecasting and Conditional Projections Using Realistic Prior Distributions. Econometric Reviews 1984; 3: 1-100.

Dua P and Ray SC. A BVAR Model for the Connecticut Economy. Journal of Forecasting 1995; 14 : 167-180.

Forni M, Hallin M, Lippi M and Reichlin L. The Generalized Dynamic Factor Model: identification and estimation. Review of Economics and Statistics 2000; 82: 540-554.

Forni M, Hallin M, Lippi M and Reichlin L. Coincident and Leading Indicators for the Euro Area. The Economic Journal 2001, 111: 62-85.

Forni M, Hallin M, Lippi M and Reichlin L. The Generalized Dynamic Factor Model, One Sided Estimation and Forecasting. Journal of the American Statistical Association 2005; 100: 830-840.

Gali J. and Monacelli T. Monetary policy and exchange rate volatility in a small open Economy.

Review of Economic Studies 2005; 72:707-734.

Geweke J. The dynamic factor analysis of economic time series. In Latent variables in socio-economic models 1977 (Aigner, and A. Goldberger, eds.) Amsterdam: North Holland, 365-383.

Giannone D and Matheson TD. A New Core Inflation Indicator for New Zealand. International Journal of Central Banking 2007; 3: 145-180.

Gupta R. Forecasting the South African Economy with VARs and VECMs. South African Journal of Economics 2006; 74: 611-628.

Gupta R. Forecasting the South African Economy with Gibbs Sampled BVECMs. South African Journal of Economics 2007a, 75: 631-643.

Gupta R. Bayesian Methods of Forecasting Inventory Investment in South Africa. Forthcoming South African Journal of Economics 2008.

Gupta R and Das S. Spatial Bayesian Methods for Forecasting House Prices in Six Metropolitan Areas of South Africa. South African Journal of Economics 2008; 76: 298-313.

Gupta R and Kabundi A. A Dynamic Factor Model for Forecasting Macroeconomic Variables in South Africa”, Working Paper 2008a; 200815 , University of Pretoria, Department of Economics.

Gupta R and Kabundi A. Forecasting Macroeconomic Variables Using Large Datasets: Dynamic Factor Model versus Large-Scale BVARs. Working Paper 2008b; 200816, University of Pretoria, Department of Economics. .

Gupta R and Sichei M. A BVAR Model for the South African Economy. South African Journal of Economics 2006; 74: 391-409.

Kinal T and Ratner JA. VAR Forecasting Model of a Regional Economy: Its Construction and Comparison. International Regional Science Review 1986, 10: 113-126.

Kwiatowski D, Phillips PCB, Schmidt P, and Shin Y. Testing the Null Hypothesis of Stationarity Against the Alternative of a Unit Root: How Sure Are We That Economic Time Series Have a Unit Root?” Journal of Econometrics 1992; 54: 159-178.

LeSage JP. A Comparison of the Forecasting Ability of ECM and VAR Models. The Review of Economics and Statistics 1990; 72:664-671.

LeSage JP. Applied Econometrics Using MATLAB, www.spatial-econometrics.com. 1999. 
Litterman RB. A Bayesian Procedure for Forecasting with Vector Autoregressions. Working Paper 1981, Federal Reserve Bank of Minneapolis.

Litterman RB. Forecasting with Bayesian Vector Autoregressions - Five Years of Experience. Journal of Business and Economic Statistics 1986; 4:25-38.

Liu G, Gupta R and Schaling E. A New Keynesian DSGE Model for forecasting the South African Economy. Forthcoming Journal of Forecasting 2008.

Lubik $\mathrm{T}$ and Schorfheide F. Do central banks respond to exchange rate movements? A structural investigation. Journal of Monetary Economics 2007; 54: 1069-1087.

Lucas RE Jr., Econometric Policy Evaluation: A Critique. Carnegie Rochester Conference Series on Public Policy 1976; 1: 19-46.

Ortiz A and Sturzenegger F. Estimating SARB's Policy Reaction Rule. South African Journal of Economics 2007; 75: 659-680.

Sargent TJ and Sims CA. Business cycle modelling without pretending to have too much a priori economic theory. In New methods in business research 1977 (C. Sims, eds.) Federal Reserve Bank of Minneapolis.

Shoesmith GL. Co-integration, Error Correction and Medium-Term Regional VAR Forecasting. Journal of Forecasting 1992; 11: 91-109.

Sims CA. Stock JH and Watson MW. Inference in Linear Time Series Models with Some Unit Roots. Econometrica 1990; 58: 113-144.

Sims CA. Macroeconomics and Reality. Econometrica 1980; 48:1-48.

Spencer DE. Developing a Bayesian Vector Autoregression Model. International Journal of Forecasting 1993, 9: 407-421.

Stock JH and Watson MW. New indexes of coincident and leading economic indicators. NBER Macroeconomics Annual1989; 351-393.

Stock JH and Watson MW. A probability model of the coincident indicators. In Leading economic indicators: New approaches and forecasting record 1991 (K. Lahiri, and G. Moore, eds.) Cambridge: Cambridge University Press, pp. 63-95.

Stock JH and Watson MW. Forecasting Inflation. Journal of Monetary Economics 1999; 44: 293-335.

Stock JH and Watson MW. Forecasting Using Principal Components from a Large Number of Predictors. Journal of the American Statistical Association 2002a; 97: 147-162.

Stock JH and Watson MW. Macroeconomic Forecasting Using Diffusion Indexes. Journal of Business and Economics Statistics 2002b; 20: 147-162.

Stock JH and Watson MW. Implications of Dynamic Factor Models for VAR Analysis. NBER Working Paper 2005; 11467.

Theil H. Principles of Econometrics. John Wiley: New York 1971.

Todd RM. Improving Economic Forecasting with Bayesian Vector Autoregression. Quarterly Review 1984, Federal Reserve Bank of Minneapolis, Fall, 18-29.

Van Nieuwenhuyze C. A Generalized Dynamic Factor Model for the Belgian Economy Identification of the Business Cycle and GDP Growth Forecasts. Journal of Business Cycle Measurement and Analysis 2007; 2: 213-248.

Zellner A. A Tale of Forecasting 1001 Series: The Bayesian Knight Strikes Again. International Journal of Forecasting 1986; 2:494-494.

Zita SE, and Gupta R. Modelling and Forecasting the Metical-Rand Exchange Rate. Forthcoming ICFAI Journal of Monetary Economics 2008. 
Table 1: RMSEs for Per Capita Growth (2003:01-2006:04)

\begin{tabular}{lllllll}
\hline \hline & QA & $\mathbf{1}$ & $\mathbf{2}$ & $\mathbf{3}$ & $\mathbf{4}$ & Average \\
\hline & SONKDSGE & 2.2109 & 1.5174 & 1.3121 & 1.2867 & 1.5818 \\
\hline & DFM & 1.2479 & 1.2288 & 1.053 & 1.3337 & 1.2159 \\
\hline \multirow{2}{*}{$\mathbf{w = 0 . 3 , d = 0 . 5}$} & VAR & 0.5851 & 0.6317 & 0.7127 & 0.7715 & 0.6752 \\
\cline { 2 - 7 } & BVAR(S) & 0.5235 & 0.5229 & 0.5706 & 0.6887 & $\mathbf{0 . 5 7 6 4}$ \\
\hline \multirow{2}{*}{$\mathbf{w = 0 . 2 , d = 1}$} & BVAR(L) & 0.0686 & 0.1261 & 0.0074 & 0.0627 & 0.0662 \\
\cline { 2 - 7 } $\mathbf{w}=\mathbf{0 . 1 , d = 1}$ & BVAR(L) & 0.0337 & 0.1758 & 0.0757 & 0.0398 & 0.0813 \\
\hline \multirow{2}{*}{$\mathbf{w = 0 . 2 , d = 2}$} & BVAR(S) & 1.0338 & 0.9984 & 1.0059 & 1.0815 & 1.0299 \\
\cline { 2 - 7 } & BVAR(L) & 0.0316 & 0.1613 & 0.0922 & 0.0423 & 0.0819 \\
\hline \multirow{2}{*}{$\mathbf{w = 0 . 1 , d = 2}$} & BVAR(S) & 1.0085 & 1.0310 & 1.0524 & 1.1312 & 1.0558 \\
\cline { 2 - 7 } & BVAR(L) & 0.0078 & 0.1369 & 0.0506 & 0.0396 & $\mathbf{0 . 0 5 8 7}$ \\
\hline \hline \multirow{2}{*}{$\mathbf{N o t e s : ~ Q A : ~}$} & QVAR(L) & 1.1310 & 1.0987 & 1.1101 & 1.1636 & 1.1258 \\
\hline
\end{tabular}

Notes: QA: Quarters Ahead; BVAR(S): Small-Scale BVAR; BVAR(L): Large-Scale BVAR.

Table 2: RMSEs for CPI Inflation (2003:01-2006:04)

\begin{tabular}{lllllll}
\hline \hline & QA & $\mathbf{1}$ & $\mathbf{2}$ & $\mathbf{3}$ & $\mathbf{4}$ & Average \\
\hline & SONKDSGE & 10.7136 & 10.0648 & 9.4701 & 8.1346 & 9.5958 \\
\hline & DFM & 6.1856 & 9.9879 & 13.6062 & 13.3206 & 10.7751 \\
\hline \multirow{2}{*}{$\mathbf{w = 0 . 3 , d = 0 . 5}$} & VAR & 2.7881 & 4.1060 & 4.5577 & 4.5720 & 4.0059 \\
\cline { 2 - 7 } $\mathbf{w}=\mathbf{0 . 2 , d = 1}$ & BVAR(S) & 2.8041 & 4.1013 & 4.4749 & 4.4619 & $\mathbf{3 . 9 6 0 5}$ \\
\hline \multirow{2}{*}{$\mathbf{w = 0 . 1 , d = 1}$} & BVAR(L) & 1.2349 & 1.2496 & 2.4090 & 3.4792 & $\mathbf{2 . 0 9 3 2}$ \\
\cline { 2 - 7 } & BVAR(L) & 3.0139 & 4.1096 & 4.4846 & 4.5670 & 4.0438 \\
\cline { 2 - 6 } $\mathbf{w = 0 . 2 , d = 2}$ & BVAR(S) & 1.0500 & 1.3637 & 2.8961 & 3.3117 & 2.1554 \\
\cline { 2 - 6 } & BVAR(L) & 1.2353 & 4.1741 & 4.4986 & 4.6313 & 4.1348 \\
\cline { 2 - 6 } $\mathbf{w = 0 . 1 , d = 2}$ & BVAR(L) & 3.0794 & 4.1459 & 4.6215 & 4.8613 & 4.1770 \\
\cline { 2 - 6 } & BVAR(S) & 3.2699 & 4.2006 & 4.5538 & 4.7349 & 4.1898 \\
\hline \hline
\end{tabular}

Notes: QA: Quarters Ahead; BVAR(S): Small-Scale BVAR; BVAR(L): Large-Scale BVAR. 
Table 3: RMSEs for Money Market Rate (2003:01-2006:04)

\begin{tabular}{lllllll}
\hline \hline & QA & $\mathbf{1}$ & $\mathbf{2}$ & $\mathbf{3}$ & $\mathbf{4}$ & Average \\
\hline & SONKDSGE & 7.5557 & 7.4517 & 7.4525 & 6.9300 & 7.3475 \\
\hline & DFM & 3.7583 & 5.32881 & 6.8937 & 9.7891 & 6.4425 \\
\hline & VAR & 1.3135 & 2.8008 & 4.4624 & 5.8660 & 3.6107 \\
\hline \multirow{2}{*}{$\mathbf{w}=\mathbf{0 . 3 , d = 0 . 5}$} & BVAR(S) & 1.0396 & 2.2496 & 3.5061 & 4.6329 & 2.8570 \\
\cline { 2 - 7 } $\mathbf{w}=\mathbf{0 . 2 , d = 1}$ & BVAR(L) & 0.1056 & 0.5333 & 1.6328 & 3.0729 & $\mathbf{1 . 3 3 6 1}$ \\
\hline \multirow{2}{*}{$\mathbf{w = 0 . 1 , d = 1}$} & BVAR(S) & 0.9417 & 1.9649 & 2.9883 & 3.9090 & 2.4510 \\
\hline \multirow{2}{*}{$\mathbf{w = 0 . 2 , d = 2}$} & BVAR(L) & 0.0631 & 0.5236 & 1.7007 & 3.1278 & 1.3538 \\
\hline \multirow{2}{*}{$\mathbf{w = 0 . 1 , d = 2}$} & BVAR(S) & 0.9260 & 1.8402 & 2.6982 & 3.4515 & 2.2290 \\
\hline \hline & BVAR(L) & 0.1040 & 0.6075 & 1.7581 & 3.1176 & 1.3968 \\
\hline \hline & BVAR(S) & 0.8632 & 1.7040 & 2.4573 & 3.0977 & 2.0306 \\
\hline & BVAR(S) & 0.0291 & 0.5558 & 1.8639 & 3.2962 & 1.4362 \\
\hline
\end{tabular}

Notes: QA: Quarters Ahead; BVAR(S): Small-Scale BVAR; BVAR(L): Large-Scale BVAR.

Table 4: RMSEs for Growth of Nominal Effective Exchange Rate (2003:01-2006:04)

\begin{tabular}{lllllll}
\hline \hline & QA & $\mathbf{1}$ & $\mathbf{2}$ & $\mathbf{3}$ & $\mathbf{4}$ & Average \\
\hline & SONKDSGE & 5.6498 & 5.2368 & 5.1672 & 5.3588 & $\mathbf{5 . 3 5 3 2}$ \\
\hline & DFM & 45.0636 & 41.2822 & 32.3305 & 34.8166 & 38.3732 \\
\hline \multirow{2}{*}{$\mathbf{w = 0 . 3 , d = 0 . 5}$} & VAR & 6.7466 & 7.4535 & 7.1799 & 6.9594 & 7.0849 \\
\cline { 2 - 7 } & BVAR(S) & 6.0516 & 6.5777 & 6.4149 & 6.2175 & 6.3154 \\
\hline \multirow{2}{*}{$\mathbf{w = 0 . 2 , d = 1}$} & BVAR(L) & 4.6223 & 16.5174 & 12.8449 & 17.8902 & 12.9687 \\
\hline \multirow{2}{*}{$\mathbf{w = 0 . 1 , d = 1}$} & BVAR(S) & 5.7568 & 6.0506 & 5.9232 & 5.7861 & 5.8792 \\
\hline \multirow{2}{*}{$\mathbf{w = 0 . 2 , d = 2}$} & BVAR(L) & 8.4419 & 11.3937 & 10.2379 & 14.4618 & 11.1338 \\
\cline { 2 - 7 } & BVAR(S) & 5.3985 & 5.5239 & 5.7868 & 5.4942 & $\mathbf{5 . 5 5 0 9}$ \\
\hline \multirow{2}{*}{$\mathbf{w = 0 . 1 , d = 2}$} & BVAR(L) & 9.9391 & 10.3474 & 11.9508 & 13.7865 & 11.5060 \\
\cline { 2 - 7 } & BVAR(L) & 5.8387 & 6.0363 & 5.9239 & 5.7961 & 5.8988 \\
\hline \hline
\end{tabular}

Notes: QA: Quarters Ahead; BVAR(S): Small-Scale BVAR; BVAR(L): Large-Scale BVAR. 
Table 5: Diebold-Mariano (1995) Tests (2003:1-2006:4)

\begin{tabular}{|c|c|c|c|c|}
\hline & \multicolumn{3}{|c|}{ Quarters Ahead } & \multirow[b]{2}{*}{4} \\
\hline Models & 1 & 2 & 3 & \\
\hline \multicolumn{5}{|l|}{ Per Capita Growth } \\
\hline $\begin{array}{c}\text { BVAR(L) }[\mathrm{w}=0.2, \mathrm{~d}=2] \\
\text { vs } \\
\text { BVAR(S) }[\mathrm{w}=0.3, \mathrm{~d}=0.5]\end{array}$ & $-6.9700 * * *$ & $-5.9263 * * *$ & $-6.8026 * * *$ & $-9.1415^{* * *}$ \\
\hline \multicolumn{5}{|l|}{ CPI Inflation } \\
\hline $\begin{array}{c}\text { BVAR(L) }[\mathrm{w}=0.3, \mathrm{~d}=0.5] \\
\text { vs } \\
\text { BVAR(S) }[\mathrm{w}=0.3, \mathrm{~d}=0.5]\end{array}$ & $-2.3801 * *$ & $-5.2382 * * *$ & $-4.6121^{* * *}$ & $-2.3041 * *$ \\
\hline \multicolumn{5}{|l|}{ Money Market Rate } \\
\hline $\begin{array}{c}\text { BVAR(L) }[w=0.3, d=0.5] \\
\text { vs } \\
\text { BVAR(S) }[w=0.1, d=2]\end{array}$ & $-3.4747 * * *$ & $-2.3983 * *$ & $-2.0381 * *$ & -1.3809 \\
\hline \multicolumn{5}{|c|}{ Growth of Nominal Effective Exchange Rate } \\
\hline $\begin{array}{c}\text { BVAR(L) }[\mathrm{w}=0.2, \mathrm{~d}=2] \\
\text { vs } \\
\text { BVAR(S) }[\mathrm{w}=0.1, \mathrm{~d}=1]\end{array}$ & $2.913 * * *$ & $-1.8031^{*}$ & -1.5125 & -1.3469 \\
\hline $\begin{array}{c}\text { BVAR(L) }[\mathrm{w}=0.2, \mathrm{~d}=2] \\
\text { vs } \\
\text { SONKDSGE }\end{array}$ & $2.9159 * * *$ & $-1.8041^{*}$ & -1.5128 & -1.3472 \\
\hline
\end{tabular}

